\title{
Toeplitz Operators on the Weighted Bergman Space over the Two-Dimensional Unit Ball
}

\author{
Alma García and Nikolai Vasilevski \\ Department of Mathematics, CINVESTAV, Mexico City 07360, Mexico \\ Correspondence should be addressed to Nikolai Vasilevski; nvasilev@math.cinvestav.mx
}

Received 4 December 2014; Accepted 21 January 2015

Academic Editor: Lars E. Persson

Copyright (C) 2015 A. García and N. Vasilevski. This is an open access article distributed under the Creative Commons Attribution License, which permits unrestricted use, distribution, and reproduction in any medium, provided the original work is properly cited.

We extend the known results on commutative Banach algebras generated by Toeplitz operators with radial quasi-homogeneous symbols on the two-dimensional unit ball. Spherical coordinates previously used hid a possibility to detect an essentially wider class of symbols that can generate commutative Banach Toeplitz operator algebras. We characterize these new algebras describing their properties and, under a certain extra condition, construct the corresponding Gelfand theory.

\section{Introduction}

After a detailed study of the commutative $C^{*}$-algebras generated by Toeplitz operators acting on the weighted Bergman spaces on the unit ball $[1,2]$ it was quite unexpectedly observed [3] that, contrary to the one-dimensional case of the unit disk, there exist many other Banach (not $C^{*}$ !) algebras generated by Toeplitz operators that are commutative on each weighted Bergman space. They were generated by Toeplitz operators with radial and the so-called quasi-homogeneous symbols, and the commutativity of the corresponding algebras was established just by an observation that the generating operators commute among themselves. Then the problem of constructing the Gelfand theory for these algebras emerged, being a tool to describe the properties, in particular spectral ones, of the operators forming the algebra. For the twodimensional ball, the case relevant to this paper, this step was done in [4].

Note that the Introduction of [4] stated that the paper studies the unique commutative Toeplitz operator Banach algebra on the two-dimensional ball. This was indeed completely true for the only known by that time generating radial quasi-homogeneous symbols. As it later turns out (and this is exactly what this paper is about) the spherical coordinates used in [4] hide the possibility to detect many other commutative Banach algebras on the two-dimensional ball generated by Toeplitz operators with symbols of a more general type.
In this paper we use another representation of the points of the two-dimensional ball, which permits us to extend essentially the previous class of quasi-homogeneous symbols. Instead of a very specific function $q(\theta)=\cos \theta \sin \theta$ of [4], we are dealing here with arbitrary $q(\theta) \in L_{\infty}[0, \pi / 2]$. Each commutative Banach algebra $\mathscr{T}_{\text {rad }, q, k}$, considered in the paper, is generated by Toeplitz operators with $L_{\infty}$ radial symbols and by the Toeplitz operator with symbol $q(\theta)\left(t_{1} \bar{t}_{2}\right)^{k}$. That is, the whole variety of our algebras is parametrized by $q(\theta) \in L_{\infty}[0, \pi / 2]$ and $k \in \mathbb{N}$.

All these algebras share many common properties. We discuss, in particular, the description of the invariant subspaces, the property of being not semisimple, radical elements description, and non-uniqueness of the representation of elements in a dense subalgebra.

The tools that we use for the explicit description of both the compact set of maximal ideals of our algebras $\mathscr{T}_{\text {rad, } q, k}$ and the Gelfand transform require the continuity of $q(\theta)\left(t_{1} \bar{t}_{2}\right)^{k}$ on the boundary of the unit ball. Thus here we impose an extra condition: $q(\theta) \in C[0, \pi / 2]$ and $q(0)=q(\pi / 2)=0$ (which is obviously satisfied in the particular case of $q(\theta)=\cos \theta \sin \theta$ in [4]).

\section{Preliminaries}

2.1. Definitions and Basic Properties. Let $\mathbb{B}^{2}$ be the open unit ball in $\mathbb{C}^{2}$ and let $d v$ denote the standard volume form on $\mathbb{B}^{2}$. 
For $\lambda>-1$, we introduce the one-parameter family of the standard weighted (probability) measures

$$
d v_{\lambda}(z)=\frac{\Gamma(\lambda+3)}{\pi^{2} \Gamma(\lambda+1)}\left(1-|z|^{2}\right)^{\lambda} d \nu(z)
$$

The weighted Bergman space $\mathscr{A}_{\lambda}^{2}\left(\mathbb{B}^{2}\right)$ is the closed subspace of $L_{2}\left(\mathbb{B}^{2}, d v_{\lambda}\right)$ that consists of all analytic functions. The orthogonal Bergman projection $B_{\lambda}$ of $L_{2}\left(\mathbb{B}^{2}, d \nu_{\lambda}\right)$ onto $\mathscr{A}_{\lambda}^{2}\left(\mathbb{B}^{2}\right)$ has the form

$$
\left(B_{\lambda} f\right)(z)=\int_{\mathbb{B}^{2}} \frac{f(\zeta) d \nu_{\lambda}(\zeta)}{(1-\langle z, \zeta\rangle)^{\lambda+3}}
$$

The reproducing kernel of $\mathscr{A}_{\lambda}^{2}\left(\mathbb{B}^{2}\right)$ is defined by

$$
K_{z}^{\lambda}(w)=\frac{1}{(1-\langle w, z\rangle)^{\lambda+3}}=\sum_{|\alpha|=0}^{\infty} \frac{\Gamma(|\alpha|+\lambda+3)}{\alpha ! \Gamma(\lambda+3)} \bar{z}^{\alpha} w^{\alpha} .
$$

The standard orthonormal monomial basis $\left\{e_{\alpha}: \alpha \in \mathbb{Z}_{+}^{2}\right\}$ of $\mathscr{A}_{\lambda}^{2}\left(\mathbb{B}^{2}\right)$ is of the form

$$
e_{\alpha}(z):=\sqrt{\frac{\Gamma(|\alpha|+\lambda+3)}{\alpha ! \Gamma(\lambda+3)}} z^{\alpha}
$$

Given a function $a \in L_{\infty}\left(\mathbb{B}^{2}\right)$, the Toeplitz operator $T_{a}$ with symbol $a$ and acting on $\mathscr{A}_{\lambda}^{2}\left(\mathbb{B}^{2}\right)$ is defined by

$$
T_{a} f=B_{\lambda}(a f), \quad f \in \mathscr{A}_{\lambda}^{2}\left(\mathbb{B}^{2}\right) .
$$

Recall also [4] that, given a radial function $a=a(r) \epsilon$ $L_{\infty}(0,1)$, the Toeplitz operator $T_{a}$ is diagonal with respect to the monomial basis (4) and its eigenvalue sequence is given by

$$
\begin{aligned}
\gamma_{a, \lambda}(\alpha)= & \frac{\Gamma(|\alpha|+\lambda+3)}{\Gamma(\lambda+1) \Gamma(|\alpha|+2)} \\
& \cdot \int_{0}^{1} a(\sqrt{r})(1-r)^{\lambda} r^{|\alpha|+1} d r, \quad \alpha \in \mathbb{Z}_{+}^{2} .
\end{aligned}
$$

In order to define our class of symbols we start with some notation.

We write a point $z \in \mathbb{B}^{2}$ as $z=\left(z_{1}, z_{2}\right), z_{j} \in \mathbb{C}$. Given a multi-index $\alpha=\left(\alpha_{1}, \alpha_{2}\right)$, we use the standard notation $z^{\alpha}=$ $z_{1}^{\alpha_{1}} z_{2}^{\alpha_{2}}$

We represent each coordinate of $z \in \mathbb{B}^{2}$ in the form $z_{j}=$ $r_{j} t_{j}$, where

$$
r_{j}=\left|z_{j}\right|, \quad t_{j}=\frac{z_{j}}{r_{j}} \in \mathbb{T} .
$$

Then we pass from the Cartesian coordinates $r_{1}, r_{2}$ on the base $\tau\left(\mathbb{B}^{2}\right)$ of the unit ball $\mathbb{B}^{2}$ to the polar coordinates $r, \theta: r_{1}=$ $r \cos \theta$, and $r_{2}=r \sin \theta$, with $r \in[0,1)$ and $\theta \in[0, \pi / 2]$.

The Toeplitz operators with radial quasi-homogeneous symbols on the two-dimensional ball were studied in detail in [4]. The quasi-homogeneous part of the symbols consisted of the following functions:

$$
\left(\frac{r_{1} r_{2}}{r_{1}^{2}+r_{2}^{2}} t_{1} \bar{t}_{2}\right)^{k}, \quad k \in \mathbb{N}
$$

Here we extend this class of symbols to the functions of the form

$$
q(\theta)\left(t_{1} \bar{t}_{2}\right)^{k}, \quad q(\theta) \in L_{\infty}\left[0, \frac{\pi}{2}\right], k \in \mathbb{N} \text {. }
$$

Note that the old functions (8) correspond to the very particular case of (9):

$$
q(\theta)=(\cos \theta \cdot \sin \theta)^{k}
$$

To start our analysis we consider the action of $T_{q(\theta)\left(t_{1} \overline{t_{2}}\right)^{k}}$ on monomials:

$$
\begin{aligned}
\left\langle T_{q\left(t_{1} \overline{z_{2}}\right)} z^{\alpha}, z^{\beta}\right\rangle & \left\langle q\left(t_{1} \overline{t_{2}}\right)^{k} z^{\alpha}, z^{\beta}\right\rangle \\
= & \frac{\Gamma(\lambda+3)}{\pi^{2} \Gamma(\lambda+1)} \\
& \cdot \int_{\mathbb{B}^{2}} q\left(1-r^{2}\right)^{\lambda} r_{1}^{\alpha_{1}+\beta_{1}+1} r_{2}^{\alpha_{2}+\beta_{2}+1} \\
= & \frac{\Gamma(\lambda+3)}{\pi^{2} \Gamma(\lambda+1)} \int_{\tau\left(\mathbb{B}^{2}\right)} q\left(1-r^{2}\right)^{\lambda} r_{1}^{\alpha_{1}+\beta_{1}+1} r_{2}^{\alpha_{2}+\beta_{2}+1} d r_{1} d r_{2} \\
& \cdot \int_{0}^{2 \pi} e^{i\left(\alpha_{1}-\beta_{1}+k\right) \theta_{1}} e^{i\left(\alpha_{2}-\beta_{2}-k\right) \theta_{2}} d r_{1} d \theta_{1} \int_{0}^{2 \pi} e^{i\left(\alpha_{2}-\beta_{2}-k\right) \theta_{2}} d \theta_{2} \\
= & \left\{\begin{array}{l}
c_{q}(\alpha), \quad \text { if } \beta=\left(\alpha_{1}+k, \alpha_{2}-k\right), \\
0, \quad \text { otherwise, }
\end{array}\right.
\end{aligned}
$$

where (making the change of variables $r_{1}=r \cos \theta, r_{2}=$ $r \sin \theta)$

$$
\begin{aligned}
c_{q}(\alpha)= & \frac{2^{2} \Gamma(\lambda+3)}{\Gamma(\lambda+1)} \\
& \cdot \int_{\tau\left(\mathbb{B}^{2}\right)} q\left(1-r^{2}\right)^{\lambda} r_{1}^{2 \alpha_{1}+k+1} r_{2}^{2 \alpha_{2}-k+1} d r_{1} d r_{2} \\
= & \frac{2^{2} \Gamma(\lambda+3)}{\Gamma(\lambda+1)} \int_{0}^{1}\left(1-r^{2}\right)^{\lambda} r^{2|\alpha|+3} d r \\
& \cdot \int_{0}^{\pi / 2} q(\theta)(\cos \theta)^{2 \alpha_{1}+k+1}(\sin \theta)^{2 \alpha_{2}-k+1} d \theta
\end{aligned}
$$




$$
\begin{aligned}
= & \frac{2 \Gamma(\lambda+3)}{\Gamma(\lambda+1)} \int_{0}^{1}(1-r)^{\lambda} r^{|\alpha|+1} d r \\
& \cdot \int_{0}^{1} q(\arccos s) s^{2 \alpha_{1}+k+1}\left(1-s^{2}\right)^{\alpha_{2}-k / 2} d s \\
= & \frac{\Gamma(\lambda+3)}{\Gamma(\lambda+1)} \frac{\Gamma(\lambda+1) \Gamma(|\alpha|+2)}{\Gamma(|\alpha|+\lambda+3)} \\
& \cdot \int_{0}^{1} q(\arccos \sqrt{s}) s^{\alpha_{1}+k / 2}(1-s)^{\alpha_{2}-k / 2} d s \\
= & \frac{\Gamma(\lambda+3) \Gamma(|\alpha|+2)}{\Gamma(|\alpha|+\lambda+3)} \\
& \cdot \int_{0}^{1} q(\arccos \sqrt{s}) s^{\alpha_{1}+k / 2}(1-s)^{\alpha_{2}-k / 2} d s .
\end{aligned}
$$

Taking into account that

$$
\left\langle z^{\beta}, z^{\beta}\right\rangle=\frac{\Gamma(\lambda+3) \Gamma\left(\beta_{1}+1\right) \Gamma\left(\beta_{2}+1\right)}{\Gamma(|\beta|+\lambda+3)},
$$

we come to the following result.

Lemma 1. Given $q=q(\theta) \in L_{\infty}[0, \pi / 2]$, one has

$$
T_{q\left(t_{1} \bar{\tau}_{2}\right)} z_{1}^{\alpha_{1}} z_{2}^{\alpha_{2}}=\widetilde{\gamma}_{q}(\alpha) z_{1}^{\alpha_{1}+k} z_{2}^{\alpha_{2}-k}, \quad \alpha \in \mathbb{Z}_{+}^{2}, \alpha_{2} \geq k,
$$

where

$$
\begin{aligned}
\tilde{\gamma}_{q}(\alpha)= & \frac{\Gamma(|\alpha|+2)}{\Gamma\left(\alpha_{1}+k+1\right) \Gamma\left(\alpha_{2}-k+1\right)} \\
& \cdot \int_{0}^{1} q(\arccos \sqrt{s}) s^{\alpha_{1}+k / 2}(1-s)^{\alpha_{2}-k / 2} d s .
\end{aligned}
$$

Corollary 2. The action of $T_{q\left(t_{1} \bar{t}_{2}\right)^{k}}$ does not depend on the weight parameter $\lambda$.

Consider now some particular cases of $q$ with $k=1$.

(i) Let $q \equiv 1$; that is, we deal with the operator $T_{t_{1} \bar{t}_{2}}$; then

$$
\begin{aligned}
\tilde{\gamma}_{1}(\alpha) & =\frac{\Gamma(|\alpha|+2)}{\Gamma\left(\alpha_{1}+2\right) \Gamma\left(\alpha_{2}\right)} \frac{\Gamma\left(\alpha_{1}+(3 / 2)\right) \Gamma\left(\alpha_{2}+(1 / 2)\right)}{\Gamma(|\alpha|+2)} \\
& =\frac{\Gamma\left(\alpha_{1}+(3 / 2)\right) \Gamma\left(\alpha_{2}+(1 / 2)\right)}{\Gamma\left(\alpha_{1}+2\right) \Gamma\left(\alpha_{2}\right)} .
\end{aligned}
$$

(ii) Let $q=\cos \theta \sin \theta$; that is, we deal with the operator $T_{\cos \theta \sin \theta t_{1} \bar{t}_{2}} ;$ then

$$
\begin{aligned}
\tilde{\gamma}_{q}(\alpha) & =\frac{\Gamma(|\alpha|+2)}{\Gamma\left(\alpha_{1}+2\right) \Gamma\left(\alpha_{2}\right)} \int_{0}^{1} s^{\alpha_{1}+1}(1-s)^{\alpha_{2}} d s \\
& =\frac{\Gamma(|\alpha|+2)}{\Gamma\left(\alpha_{1}+2\right) \Gamma\left(\alpha_{2}\right)} \cdot \frac{\Gamma\left(\alpha_{1}+2\right) \Gamma\left(\alpha_{2}+1\right)}{\Gamma(|\alpha|+3)} \\
& =\frac{\alpha_{2}}{|\alpha|+2} .
\end{aligned}
$$

This is a particular case of (8) which was studied in [4].

Take now any $a=a(r)$ and $q=q(\theta)$, both from $L_{\infty}$, and $k \in \mathbb{N}$; then

$$
\left\langle T_{a q\left(t_{1} \bar{t}_{2}\right)^{k}} z^{\alpha}, z^{\beta}\right\rangle=0, \quad \forall \beta \neq\left(\alpha_{1}+k, \alpha_{2}-k\right) .
$$

Thus we calculate

$$
\begin{aligned}
& \left\langle T_{a q\left(t_{1} \bar{t}_{2}{ }^{k}{ }^{\alpha}\right.} z^{\alpha} z_{1}^{\alpha_{1}+k} z_{2}^{\alpha_{2}-k}\right\rangle \\
& =\frac{2^{2} \Gamma(\lambda+3)}{\Gamma(\lambda+1)}
\end{aligned}
$$

$$
\begin{aligned}
& \cdot \int_{\tau\left(\mathbb{B}^{2}\right)} a q\left(1-r^{2}\right)^{\lambda} r_{1}^{2 \alpha_{1}+k+1} r_{2}^{2 \alpha_{2}-k+1} d r_{1} d r_{2} \\
= & \frac{2^{2} \Gamma(\lambda+3)}{\Gamma(\lambda+1)} \int_{0}^{1} a(r)\left(1-r^{2}\right)^{\lambda} r^{2|\alpha|+3} d r \\
& \cdot \int_{0}^{\pi / 2} q(\theta)(\cos \theta)^{2 \alpha_{1}+k+1}(\sin \theta)^{2 \alpha_{2}-k+1} d \theta \\
= & \frac{2 \Gamma(\lambda+3)}{\Gamma(\lambda+1)} \int_{0}^{1} a(\sqrt{r})(1-r)^{\lambda} r^{|\alpha|+1} d r \\
& \cdot \int_{0}^{1} q(\arccos s) s^{2 \alpha_{1}+k+1}\left(1-s^{2}\right)^{\alpha_{2}-k / 2} d s \\
= & \frac{\Gamma(|\alpha|+\lambda+3)}{\Gamma(\lambda+1) \Gamma(|\alpha|+2)} \int_{0}^{1} a(\sqrt{r})(1-r)^{\lambda} r^{|\alpha|+1} d r \\
& \cdot \frac{\Gamma(\lambda+3) \Gamma(|\alpha|+2)}{\Gamma(|\alpha|+\lambda+3)} \\
& \cdot \int_{0}^{1} q(\arccos \sqrt{s}) s^{\alpha_{1}+k / 2}(1-s)^{\alpha_{2}-k / 2} d s .
\end{aligned}
$$

That is,

$$
T_{a q\left(t_{1} \bar{t}_{2}\right)^{k}} z^{\alpha}=c_{a, q}(\alpha) z_{1}^{\alpha_{1}+k} z_{2}^{\alpha_{2}-k}
$$

where

$$
\begin{aligned}
& c_{a, q}(\alpha) \\
& =\frac{\Gamma(|\alpha|+\lambda+3)}{\Gamma(\lambda+3) \Gamma\left(\alpha_{1}+k+1\right) \Gamma\left(\alpha_{2}-k+1\right)}
\end{aligned}
$$




$$
\begin{aligned}
& \cdot \frac{\Gamma(|\alpha|+\lambda+3)}{\Gamma(\lambda+1) \Gamma(|\alpha|+2)} \int_{0}^{1} a(\sqrt{r})(1-r)^{\lambda} r^{|\alpha|+1} d r \\
& \cdot \frac{\Gamma(\lambda+3) \Gamma(|\alpha|+2)}{\Gamma(|\alpha|+\lambda+3)} \\
& \cdot \int_{0}^{1} q(\arccos \sqrt{s}) s^{\alpha_{1}+k / 2}(1-s)^{\alpha_{2}-k / 2} d s \\
= & \frac{\Gamma(|\alpha|+\lambda+3)}{\Gamma(\lambda+1) \Gamma(|\alpha|+2)} \int_{0}^{1} a(\sqrt{r})(1-r)^{\lambda} r^{|\alpha|+1} d r \\
& \cdot \frac{\Gamma(|\alpha|+2)}{\Gamma(\alpha+k+1) \Gamma\left(\alpha_{2}-k+1\right)} \\
& \cdot \int_{0}^{1} q(\arccos \sqrt{s}) s^{\alpha_{1}+k / 2}(1-s)^{\alpha_{2}-k / 2} d s \\
= & \gamma_{a, \lambda}(|\alpha|) \cdot \tilde{\gamma}_{q}(\alpha),
\end{aligned}
$$

or

$$
T_{a q\left(t_{1} \bar{t}_{2}\right)^{k}} z^{\alpha}=\gamma_{a, \lambda}(|\alpha|) \cdot \widetilde{\gamma}_{q}(\alpha) z_{1}^{\alpha_{1}+k} z_{2}^{\alpha_{2}-k}
$$

Note that the last formula implies the next result.

Lemma 3. Given any $a=a(r)$ and any $q=q(\theta)$, both from $L_{\infty}$, one has

$$
T_{a q\left(t_{1} \bar{t}_{2}\right)^{k}}=T_{a} \cdot T_{q\left(t_{1} \bar{t}_{2}\right)^{k}}=T_{q\left(t_{1} \bar{t}_{2}\right)^{k}} \cdot T_{a} .
$$

In what follows, to simplify the notation, we introduce the pairs $\kappa=(k,-k), k \in \mathbb{N}$, and $t=\left(t_{1}, t_{2}\right) \in \mathbb{T}^{2}$. Then

$$
\left(t_{1} \bar{t}_{2}\right)^{k}=t_{1}^{k} t_{2}^{-k}=t^{\kappa}
$$

so that $q(\theta)\left(t_{1} \bar{t}_{2}\right)^{k}=q t^{\kappa}$.

2.2. Comparison of the Toeplitz Operators with Symbols (8) and (9). Let $q_{1}, q_{2} \in L_{\infty}[0, \pi / 2], k_{1}, k_{2} \in \mathbb{N}$, and $\kappa_{1}=\left(k_{1},-k_{1}\right)$, $\kappa_{2}=\left(k_{2},-k_{2}\right)$. Consider the symbols $q_{1} t^{\kappa_{1}}$ and $q_{2} t^{\kappa_{2}}$, and calculate the action on the monomials of the products of the corresponding Toeplitz operators:

$$
\begin{aligned}
T_{q_{1} t^{\kappa_{1}}} & T_{q_{2} t^{k_{2}}} z^{\alpha} \\
= & \frac{\Gamma(|\alpha|+2)}{\Gamma\left(\alpha_{1}+k_{2}+k_{1}+1\right) \Gamma\left(\alpha_{2}-k_{2}-k_{1}+1\right)} \\
& \cdot \int_{0}^{1} q_{1}(\arccos \sqrt{s}) s^{\alpha_{1}+k_{2}+k_{1} / 2}(1-s)^{\alpha_{2}-k_{2}-k_{1} / 2} d s \\
& \cdot \frac{\Gamma(|\alpha|+2)}{\Gamma\left(\alpha_{1}+k_{2}+1\right) \Gamma\left(\alpha_{2}-k_{2}+1\right)} \\
& \cdot \int_{0}^{1} q_{2}(\arccos \sqrt{s}) s^{\alpha_{1}+k_{2} / 2}(1-s)^{\alpha_{2}-k_{2} / 2} d s \\
& \cdot z^{\left(\alpha_{1}+k_{2}+k_{1}, \alpha_{2}-k_{2}-k_{1}\right)},
\end{aligned}
$$

$$
\begin{aligned}
T_{q_{2} t^{\kappa_{2}}} T_{q_{1} t^{\kappa_{1}}} z^{\alpha} & \frac{\Gamma(|\alpha|+2)}{\Gamma\left(\alpha_{1}+k_{1}+k_{2}+1\right) \Gamma\left(\alpha_{2}-k_{1}-k_{2}+1\right)} \\
& \cdot \int_{0}^{1} q_{2}(\arccos \sqrt{s}) s^{\alpha_{1}+k_{1}+k_{2} / 2}(1-s)^{\alpha_{2}-k_{1}-k_{2} / 2} d s \\
& \cdot \frac{\Gamma(|\alpha|+2)}{\Gamma\left(\alpha_{1}+k_{1}+1\right) \Gamma\left(\alpha_{2}-k_{1}+1\right)} \\
& \cdot \int_{0}^{1} q_{1}(\arccos \sqrt{s}) s^{\alpha_{1}+k_{1} / 2}(1-s)^{\alpha_{2}-k_{1} / 2} d s \\
& \cdot z^{\left(\alpha_{1}+k_{1}+k_{2}, \alpha_{2}-k_{1}-k_{2}\right)} .
\end{aligned}
$$

That is, the Toeplitz operators $T_{q_{1} t^{\kappa_{1}}}$ and $T_{q_{2} t^{\kappa_{2}}}$ do not commute, in general.

Consider now a more specific case, close to (8):

$$
\begin{aligned}
& q_{1}(\theta)=\cos ^{m_{1}} \theta \sin ^{m_{2}} \theta, \\
& q_{2}(\theta)=\cos ^{\ell_{1}} \theta \sin ^{\ell_{2}} \theta .
\end{aligned}
$$

Then

$$
\begin{aligned}
& T_{q_{1} t^{\kappa_{1}}} T_{q_{2} t^{\kappa_{2}}} z^{\alpha} \\
& =\tilde{\gamma}_{q_{1}}\left(\alpha_{1}+k_{2}, \alpha_{2}-k_{2}\right) \\
& \cdot \widetilde{\gamma}_{q_{2}}\left(\alpha_{1}, \alpha_{2}\right) z_{1}^{\alpha_{1}+k_{2}+k_{1}} z_{2}^{\alpha_{2}-k_{2}-k_{1}} \\
& =\frac{\Gamma(|\alpha|+2)}{\Gamma\left(\alpha_{1}+k_{2}+k_{1}+1\right) \Gamma\left(\alpha_{2}-k_{2}-k_{1}+1\right)} \\
& \text {. } \int_{0}^{1} s^{\alpha_{1}+k_{2}+k_{1} / 2+m_{1} / 2}(1-s)^{\alpha_{2}-k_{2}-k_{1} / 2+m_{2} / 2} d s \\
& \frac{\Gamma(|\alpha|+2)}{\Gamma\left(\alpha_{1}+k_{2}+1\right) \Gamma\left(\alpha_{2}-k_{2}+1\right)} \\
& \cdot \int_{0}^{1} s^{\alpha_{1}+k_{2} / 2+\ell_{1} / 2}(1-s)^{\alpha_{2}-k_{2} / 2+\ell_{2} / 2} d s \\
& \cdot z_{1}^{\alpha_{1}+k_{2}+k_{1}} z_{2}^{\alpha_{2}-k_{2}-k_{1}} \\
& =\Gamma(|\alpha|+2) \Gamma\left(\alpha_{1}+k_{2}+\frac{k_{1}}{2}+\frac{m_{1}}{2}+1\right) \\
& \cdot \Gamma\left(\alpha_{2}-k_{2}-\frac{k_{1}}{2}+\frac{m_{2}}{2}+1\right) \\
& \cdot\left(\Gamma\left(\alpha_{1}+k_{2}+k_{1}+1\right) \Gamma\left(\alpha_{2}-k_{2}-k_{1}+1\right)\right. \\
& \left.\cdot \Gamma\left(|\alpha|+\frac{m_{1}+m_{2}}{2}+2\right)\right)^{-1} \\
& \cdot \Gamma\left(\alpha_{1}+\frac{k_{2}}{2}+\frac{\ell_{1}}{2}+1\right) \Gamma\left(\alpha_{2}-\frac{k_{2}}{2}+\frac{\ell_{2}}{2}+1\right)
\end{aligned}
$$




$$
\begin{aligned}
& \cdot\left(\Gamma\left(\alpha_{1}+k_{2}+1\right) \Gamma\left(\alpha_{2}-k_{2}+1\right)\right. \\
& \left.\cdot \Gamma\left(|\alpha|+\frac{\ell_{1}+\ell_{2}}{2}+2\right)\right)^{-1} \\
& \cdot z_{1}^{\alpha_{1}+k_{2}+k_{1}} z_{2}^{\alpha_{2}-k_{2}-k_{1}} \text {, } \\
& T_{q_{2} t^{\kappa_{2}}} T_{q_{1} t^{\kappa_{1}}} z^{\alpha} \\
& =\widetilde{\gamma}_{q_{2}}\left(\alpha_{1}+k_{1}, \alpha_{2}-k_{1}\right) \\
& \text { - } \widetilde{\gamma}_{q_{1}}\left(\alpha_{1}, \alpha_{2}\right) z_{1}^{\alpha_{1}+k_{1}+k_{2}} z_{2}^{\alpha_{2}-k_{1}-k_{2}} \\
& =\frac{\Gamma(|\alpha|+2)}{\Gamma\left(\alpha_{1}+k_{1}+k_{2}+1\right) \Gamma\left(\alpha_{2}-k_{1}-k_{2}+1\right)} \\
& \cdot \int_{0}^{1} s^{\alpha_{1}+k_{1}+k_{2} / 2+\ell_{1} / 2}(1-s)^{\alpha_{2}-k_{1}-k_{2} / 2+\ell_{2} / 2} d s \\
& \cdot \frac{\Gamma(|\alpha|+2)}{\Gamma\left(\alpha_{1}+k_{1}+1\right) \Gamma\left(\alpha_{2}-k_{1}+1\right)} \\
& \cdot \int_{0}^{1} s^{\alpha_{1}+k_{1} / 2+m_{1} / 2}(1-s)^{\alpha_{2}-k_{1} / 2+m_{2} / 2} d s \\
& \cdot z_{1}^{\alpha_{1}+k_{1}+k_{2}} z_{2}^{\alpha_{2}-k_{1}-k_{2}} \\
& =\Gamma(|\alpha|+2) \Gamma\left(\alpha_{1}+k_{1}+\frac{k_{2}}{2}+\frac{\ell_{1}}{2}+1\right) \\
& \cdot \Gamma\left(\alpha_{2}-k_{1}-\frac{k_{2}}{2}+\frac{\ell_{2}}{2}+1\right) \\
& \cdot\left(\Gamma\left(\alpha_{1}+k_{1}+k_{2}+1\right) \Gamma\left(\alpha_{2}-k_{1}-k_{2}+1\right)\right. \\
& \left.\cdot \Gamma\left(|\alpha|+\frac{\ell_{1}+\ell_{2}}{2}+2\right)\right)^{-1} \\
& \cdot \Gamma\left(\alpha_{1}+\frac{k_{1}}{2}+\frac{m_{1}}{2}+1\right) \\
& \cdot \Gamma\left(\alpha_{2}-\frac{k_{1}}{2}+\frac{m_{2}}{2}+1\right) \\
& \cdot\left(\Gamma\left(\alpha_{1}+k_{1}+1\right) \Gamma\left(\alpha_{2}-k_{1}+1\right)\right. \\
& \left.\cdot \Gamma\left(|\alpha|+\frac{m_{1}+m_{2}}{2}+2\right)\right)^{-1} \\
& \cdot z_{1}^{\alpha_{1}+k_{1}+k_{2}} z_{2}^{\alpha_{2}-k_{1}-k_{2}} \text {. }
\end{aligned}
$$

That is, $T_{q_{1} t^{\kappa_{1}}}$ commutes with $T_{q_{2} t^{\kappa_{2}}}$ if and only if

$$
\begin{gathered}
\Gamma\left(\alpha_{1}+k_{2}+\frac{k_{1}}{2}+\frac{m_{1}}{2}+1\right) \Gamma\left(\alpha_{2}-k_{2}-\frac{k_{1}}{2}+\frac{m_{2}}{2}+1\right) \\
\cdot \Gamma\left(\alpha_{1}+\frac{k_{2}}{2}+\frac{\ell_{1}}{2}+1\right) \Gamma\left(\alpha_{2}-\frac{k_{2}}{2}+\frac{\ell_{2}}{2}+1\right)
\end{gathered}
$$

$$
\begin{aligned}
& \cdot\left(\Gamma\left(\alpha_{1}+k_{2}+1\right) \Gamma\left(\alpha_{2}-k_{2}+1\right)\right)^{-1} \\
= & \Gamma\left(\alpha_{1}+k_{1}+\frac{k_{2}}{2}+\frac{\ell_{1}}{2}+1\right) \Gamma\left(\alpha_{2}-k_{1}-\frac{k_{2}}{2}+\frac{\ell_{2}}{2}+1\right) \\
& \cdot \Gamma\left(\alpha_{1}+\frac{k_{1}}{2}+\frac{m_{1}}{2}+1\right) \Gamma\left(\alpha_{2}-\frac{k_{1}}{2}+\frac{m_{2}}{2}+1\right) \\
& \cdot\left(\Gamma\left(\alpha_{1}+k_{1}+1\right) \Gamma\left(\alpha_{2}-k_{1}+1\right)\right)^{-1} .
\end{aligned}
$$

The last equality holds if and only if

$$
m_{1}=m_{2}=k_{1}, \quad \ell_{1}=\ell_{2}=k_{2} .
$$

This brings us back to the case analyzed in [4], showing that in our more general case of $q \in L_{\infty}[0, \pi / 2]$ we have fewer properties as compared with the specific case of [4]. In particular, we lose the commutativity of generating operators in [4], extending at the same time the class of generating symbols.

\section{The Algebra $\mathscr{T}_{\text {rad }, q, k}$}

Denote by $\mathscr{T}_{\text {rad }}$ the $C^{*}$-algebra generated by all Toeplitz operators $T_{a}$ 's with radial symbols $a=a(r) \in L_{\infty}[0,1)$. We fix then a function $q=q(\theta) \in L_{\infty}[0, \pi / 2]$ and $k \epsilon$ $\mathbb{N}$ and denote by $\mathscr{T}_{q, k}$ the unital Banach algebra generated by Toeplitz operator $T_{q t^{k}}$. Since the generators of both these algebras commute (Lemma 3), the Banach algebra $\mathscr{T}_{\text {rad, } q, k}$ generated by elements of $\mathscr{T}_{\text {rad }}$ and $\mathscr{T}_{q, k}$ is commutative.

In this section we study the algebra $\mathscr{T}_{\text {rad }, q, k}$ as well its generating subalgebras. The analysis of the algebra $\mathscr{T}_{\mathrm{rad}}$ is the same as in [4, Section 3.1]; thus we recall here just the most important facts.

3.1. Toeplitz Operators with Radial Symbols. Given a sequence $\gamma=\{\gamma(|\alpha|)\}_{|\alpha| \in \mathbb{Z}_{+}} \in \ell_{\infty}$, we denote by $D_{\gamma}$ the diagonal operator defined on $\mathscr{A}_{\lambda}^{2}\left(\mathbb{B}^{2}\right)$ as follows:

$$
D_{\gamma} z^{\alpha}=\gamma(|\alpha|) z^{\alpha}, \quad \alpha \in \mathbb{Z}_{+}^{2} .
$$

If $T_{a}$ is a Toeplitz operator with radial symbol we have obviously

$$
T_{a}=D_{\gamma_{a, \lambda}} .
$$

Recall [5, Section 5] that the sequence $\gamma_{a, \lambda}$ of a Toeplitz operator $T_{a}$ belongs to the $C^{*}$-algebra $\mathrm{SO}\left(\mathbb{Z}_{+}\right)$, where $\mathrm{SO}\left(\mathbb{Z}_{+}\right)$ consists of all bounded sequences that slowly oscillate in the sense of Schmidt [6]; that is,

$$
\operatorname{SO}\left(\mathbb{Z}_{+}\right)=\left\{x \in \ell_{\infty}: \lim _{(j+1) /(i+1) \rightarrow 1}\left|x_{j}-x_{i}\right|=0\right\} .
$$

Moreover [5, Section 5], the $C^{*}$-algebra $\mathscr{T}_{\text {rad }}$ is isomorphic and isometric to the algebra $\mathrm{SO}\left(\mathbb{Z}_{+}\right)$, via identification of a diagonal operator with its eigenvalue sequence.

Corollary 4. Let $\gamma$ be a convergent sequence; then $D_{\gamma} \in \mathscr{T}_{\mathrm{rad}}$. In particular, for all $n \in \mathbb{Z}_{+}$the orthogonal projection $P_{n}$ of $\mathscr{A}_{\lambda}^{2}\left(\mathbb{B}^{2}\right)$ onto $\operatorname{span}\left\{z^{\alpha}:|\alpha|=n\right\}$ belongs to the algebra $\mathscr{T}_{\text {rad }}$. 
Recall [4] that the compact set $M\left(\mathscr{T}_{\text {rad }}\right)$ of maximal ideals of the algebra $\mathscr{T}_{\text {rad }}$ has the form

$$
M\left(\mathscr{T}_{\text {rad }}\right)=\mathbb{Z}_{+} \cup M_{\infty} .
$$

The fiber $M_{\infty}$ is the set of all multiplicative functionals $\varphi$ such that $\varphi\left(D_{\gamma}\right)=0$ whenever $D_{\gamma}$ is a compact operator, or $\gamma \in c_{0}$, where $c_{0}$ denotes the set of all sequences converging to zero. And $\mathbb{Z}_{+}$can be considered a part of $M\left(\mathscr{T}_{\text {rad }}\right)$ since each $\ell \in$ $\mathbb{Z}_{+}$defines the multiplicative evaluation functional $\varphi_{\ell}: D_{\gamma} \mapsto$ $\gamma(\ell)$.

Moreover, by [7, Chapter I, Theorem 8.2], the set $\mathbb{Z}_{+}$ is densely and homeomorphically embedded into $M\left(\mathscr{T}_{\mathrm{rad}}\right)$, and, by [8], the fiber $M_{\infty}$ is connected.

3.2. Toeplitz Operators with Symbol $q(\theta)\left(t_{1} \overline{t_{2}}\right)^{k}$ and Its Spectrum. Recall that the spectrum of the Toeplitz operator $T_{q t^{k}}$ is independent of the weight parameter $\lambda$, and note that

$$
\begin{aligned}
\left\|T_{q t^{\kappa}}\right\| & \leq\left\|q(\theta)\left(t_{1} \overline{t_{2}}\right)^{k}\right\|_{\infty} \\
& =\sup _{\theta \in[0, \pi / 2], t_{1}, t_{2} \in \mathbb{T}}\left|q(\theta)\left(t_{1} \overline{t_{2}}\right)^{k}\right|_{\infty}=\sup _{\theta \in[0, \pi / 2]}|q(\theta)| \\
& =\|q\|_{\infty} .
\end{aligned}
$$

That is, the spectral radius of $T_{q t^{\kappa}}$ is at most $\|q\|_{\infty}$. And (Lemma 1) since the operator $T_{q t^{\kappa}}$ is not invertible, we have that

$$
0 \in \operatorname{sp} T_{q t^{\kappa}} \subset \bar{D}\left(0,\|q\|_{\infty}\right) .
$$

We denote by $\Delta(q, k)$ the set $\operatorname{sp} T_{q t^{\kappa}}$. Then the maximal ideal space $M\left(\mathscr{T}_{q, k}\right)$ of the Banach algebra $\mathscr{T}_{q, k}$ coincides with the spectrum of its generator; that is,

$$
M\left(\mathscr{T}_{q, k}\right)=\Delta(q, k)
$$

3.3. Invariant Subspaces. Given $p \in \mathbb{Z}_{+}$, we denote by $H_{p}$ the finite dimensional subspace

$$
H_{p}=\operatorname{span}\left\{e_{\alpha}: \alpha \in \mathbb{Z}_{+},|\alpha|=p\right\}
$$

It is obvious that

$$
\mathscr{A}_{\lambda}^{2}\left(\mathbb{B}^{2}\right)=\bigoplus_{p=0}^{\infty} H_{p}
$$

Observe now that each space $H_{p}$ is invariant for all operators from $\mathscr{T}_{\text {rad }, q, k}$. Moreover, by Corollary 4, each orthogonal projection $P_{p}$ onto $H_{p}$ is a diagonal operator from $\mathscr{T}_{\text {rad }}$.

Each diagonal operator $D_{\gamma}$ restricted to $H_{p}$ is the scalar operator $\gamma(p) I$, while the operator $T_{q t^{\kappa}}$ acts on $H_{p}$ as a weighted shift operator. It is also nilpotent on each $H_{p}$, since

$$
\left(\left.T_{q t^{\star}}\right|_{H_{p}}\right)^{\lfloor p / k\rfloor+1}=0
$$

The last implies that

$$
\bigoplus_{s=0}^{(p-1) k} H_{s} \subset \operatorname{ker} T_{q t^{k}}^{p}, \quad \forall p \in \mathbb{N} .
$$

\subsection{Radical}

Lemma 5. The algebra $\mathscr{T}_{\mathrm{rad}, q, k}$ is not semisimple. Its radical $\operatorname{Rad} \mathscr{T}_{\mathrm{rad}, q, k}$ contains, in particular, the operators of the form $D_{\gamma} T_{q t^{\kappa}}$, where $\gamma \in c_{0}$.

Proof. Following the proof of [4, Lemma 3.7], we only need to prove that the operator $D_{\gamma} T_{q t^{\kappa}}$ is topologically nilpotent; that is,

$$
\lim _{p \rightarrow \infty}\left\|\left(D_{\gamma} T_{q t^{\kappa}}\right)^{p}\right\|^{1 / p}=0
$$

Note that

$$
\begin{aligned}
\left(D_{\gamma} T_{q t^{\kappa}}\right)^{p} & =D_{\gamma}^{p} T_{q t^{\kappa}}^{p} \\
& =D_{\gamma}^{p} T_{q t^{\kappa}}^{p}\left(I-\left(P_{0}+\cdots+P_{(p-1) k}\right)\right) \\
& =\left[D_{\gamma}\left(I-\left(P_{0}+\cdots+P_{(p-1) k}\right)\right)\right]^{p} T_{q t^{\kappa}}^{p} .
\end{aligned}
$$

That is, since $\gamma \in c_{0}$,

$$
\begin{aligned}
\left\|\left(D_{\gamma} T_{q t^{\kappa}}\right)^{p}\right\|^{1 / p} & \leq\left\|D_{\gamma}\left(I-\left(P_{0}+\cdots+P_{(p-1) k}\right)\right)\right\| \cdot\left\|T_{q t^{\kappa}}\right\| \\
& =\sup _{\ell>p k}|\gamma(\ell)| \cdot\left\|T_{q t^{\kappa}}\right\| \underset{p \rightarrow \infty}{\longrightarrow} 0 .
\end{aligned}
$$

3.5. Dense Subalgebra in $\mathscr{T}_{\mathrm{rad}, q, k}$. The set of all operators of the form

$$
A=\sum_{p=0}^{m} D_{\gamma_{p}} T_{q t^{\kappa}}^{p}
$$

where $D_{\gamma_{p}} \in \mathscr{T}_{\text {rad }}$, constitutes the dense (nonclosed) subalgebra $\mathscr{D}=\mathscr{D}_{\text {rad }, q, k}$ of the algebra $\mathscr{T}_{\text {rad }, q, k}$. At the same time the representation of the operators from $\mathscr{D}$ in the above form is not unique.

To describe the source of such non-uniqueness, we denote by $K(p)$ the set of finite dimensional diagonal operators with eigenvalue sequences

$$
\gamma=\{\gamma(0), \gamma(1), \ldots, \gamma((p-1) k), 0,0, \ldots\},
$$

and $K(0)=\{0\}$ for $p=0$.

Lemma 6. One has

$$
\sum_{p=0}^{m} D_{\gamma_{p}} T_{q t^{\kappa}}^{p}=0
$$

if and only if $D_{\gamma_{p}} \in K(p)$, for each $p=0,1, \ldots, m$. 
Proof. The proof follows almost literally the arguments of proof [4, Lemma 3.8], given for a particular case of $q(\theta)=$ $\cos \theta \sin \theta$ and $k=1$.

The next lemma is a special case of the previous one.

Lemma 7. Let

$$
A=\sum_{j=1}^{m}\left(\prod_{\ell=1}^{m_{j}} T_{a_{j, \ell}} T_{q t^{\kappa}}^{p_{j}}\right)=0,
$$

where all $p_{j}$ are different. Then, for each $k$ at least one radial symbol $a_{j, \ell}$ is identically zero.

Proof. The previous lemma implies that if

$$
A=\sum_{j=1}^{m}\left(\prod_{\ell=1}^{m_{j}} T_{a_{j, \ell}} T_{q t^{\kappa}}^{p_{j}}\right)=0,
$$

then each diagonal operator $\prod_{\ell=1}^{m_{k}} T_{a_{j, \ell}}$ is finite dimensional. The rest of the proof follows the arguments of the proof of [4, Theorem 3.9].

As an application to the so-called zero-product problem we give the next corollary.

Corollary 8. For the operator

$$
A=\prod_{\ell=1}^{m} T_{a_{\ell}} T_{q t^{\kappa}}^{p}
$$

the following statements are equivalent:

(1) $A=0$,

(2) A is finite dimensional,

(3) At least one radial symbol $a_{\ell}$ is identically zero.

3.6. A Special Case of $q=q(\theta)$. In what follows we will assume that a function $q=q(\theta) \in L_{\infty}[0, \pi / 2]$ satisfies the extra condition:

$$
q=q(\theta) \in C\left[0, \frac{\pi}{2}\right], \quad q(0)=0=q\left(\frac{\pi}{2}\right) .
$$

We note that this is the (only) case when the homogeneous of order zero function (our generating symbol) $q t^{\kappa}=q(\theta)\left(t_{1} \bar{t}_{2}\right)^{k}$ is continuous on the boundary $S^{3}$ of the unit ball $\mathbb{B}^{2}$.

We note also that the quasi-homogeneous symbols (8) of [4] satisfy the above conditions.

Condition (50) permits us to make the statements of Section 3.2 more precise. that

First of all (50), together with the results of [9], implies

$$
\begin{aligned}
\text { ess-sp } T_{q t^{\kappa}} & =\left.\operatorname{Im}\left(q(\theta)\left(t_{1} \overline{t_{2}}\right)^{k}\right)\right|_{\theta \in[0, \pi / 2], t_{1}, t_{2} \in \mathbb{T}} \\
& =\bar{D}\left(0,\|q\|_{\infty}\right) .
\end{aligned}
$$

Therefore

$$
\operatorname{sp} T_{q t^{\kappa}}=\Delta(q, k)=\bar{D}\left(0,\|q\|_{\infty}\right)
$$

as

$$
\bar{D}\left(0,\|q\|_{\infty}\right)=\operatorname{ess}-\mathrm{sp} T_{q t^{\kappa}} \subset \operatorname{sp} T_{q t^{\kappa}} \subset \bar{D}\left(0,\|q\|_{\infty}\right),
$$

where $D(0, r)$ is the disk of radius $r$ centered at the origin.

That is, now we have that

$$
M\left(\mathscr{T}_{q, k}\right)=\bar{D}\left(0,\|q\|_{\infty}\right)
$$

Theorem 9. The Banach algebra $\mathscr{T}_{q, k}$ is isomorphic via the Gelfand transform to the algebra $C_{a}\left(\bar{D}\left(0,\|q\|_{\infty}\right)\right)$ of all functions analytic in $D\left(0,\|q\|_{\infty}\right)$ and continuous on $\bar{D}\left(0,\|q\|_{\infty}\right)$.

Proof. It practically literally follows the proof of [4, Theorem 3.6].

\section{Gelfand Theory of $\mathscr{T}_{\text {rad }, q, k}$}

4.1. Finitely Generated Subalgebras of $\mathscr{T}_{\mathrm{rad}, q, k}$. We present here relevant material from [4], slightly modified when the more general symbols (9) will be involved.

We start from a finite number of diagonal operators $D_{\gamma_{1}}, \ldots, D_{\gamma_{n}}$ on $\mathscr{A}_{\lambda}^{2}\left(\mathbb{B}^{2}\right)$ from the algebra $\mathscr{T}_{\text {rad }}$. They act on the standard monomial basis (4) as

$$
D_{\gamma_{j}} e_{\alpha}=\gamma_{j}(|\alpha|) e_{\alpha}, \quad \text { for } \alpha \in \mathbb{Z}_{+}^{2} \text {. }
$$

Denote by

$$
\mathscr{A}_{\mathrm{D}}^{*}:=\mathscr{A}\left(D_{\gamma_{1}}, \ldots, D_{\gamma_{n}}\right) \subset L\left(\mathscr{A}_{\lambda}^{2}\left(\mathbb{B}^{2}\right)\right)
$$

the unital $C^{*}$-algebra generated by elements of $\mathbf{D}=\left(D_{\gamma_{1}}\right.$, $\left.\ldots, D_{\gamma_{n}}\right)$.

Recall [10] that the joint spectrum $\sigma\left(D_{\gamma_{1}}, \ldots, D_{\gamma_{n}}\right)$ of the operators $D_{\gamma_{1}}, \ldots, D_{\gamma_{n}}$ can be identified with the maximal ideal space $M\left(\mathscr{A}_{\mathrm{D}}^{*}\right)$ of the $C^{*}$-algebra $\mathscr{A}_{\mathrm{D}}^{*}$ and it has the form

$$
\begin{aligned}
\sigma( & \left.D_{\gamma_{1}}, \ldots, D_{\gamma_{n}}\right) \\
= & \left\{\left(\mu_{1}, \ldots, \mu_{n}\right) \in \mathbb{C}^{n}\right. \\
& \left.\quad: J\left(D_{\gamma_{1}}-\mu_{1} I, \ldots, D_{\gamma_{n}}-\mu_{n} I\right) \neq \mathscr{A}_{\mathbf{D}}^{*}\right\},
\end{aligned}
$$

where $J\left(D_{\gamma_{1}}-\mu_{1} I, \ldots, D_{\gamma_{n}}-\mu_{n} I\right)$ denotes the smallest ideal in the algebra $\mathscr{A}_{\mathrm{D}}^{*}$ containing the elements $D_{\gamma_{j}}-\mu_{j} I$, for $j=$ $1, \ldots, n$.

Lemma 10 (see $\left[4\right.$, Corollary 4.11]). Let $\left(\mu_{1}, \ldots, \mu_{n}\right) \in$ $\sigma\left(D_{\gamma_{1}}, \ldots, D_{\gamma_{n}}\right)$. Then either there is $k \in \mathbb{Z}_{+}$such that $\gamma_{j}(k)=$ $\mu_{j}$ for all $j=1, \ldots, n$ or there is a sequence $\left\{m_{\ell}\right\}_{\ell} \in \mathbb{Z}_{+}$such that for all $j=1, \ldots, n$

$$
\lim _{\ell \rightarrow \infty} \gamma_{j}\left(m_{\ell}\right)=\mu_{j} .
$$


Let $\left(\mu_{1}, \ldots, \mu_{n}\right) \in \sigma\left(D_{\gamma_{1}}, \ldots, D_{\gamma_{n}}\right)$. Assuming the first option of the above lemma, we define a multiplicative functional $\psi_{(k)}$ on $\mathscr{A}_{\mathrm{D}}^{*}$ by

$$
\psi_{(k)}(D):=\left\langle D e_{(k, 0)}, e_{(k, 0)}\right\rangle
$$

for all $D \in \mathscr{A}_{\mathbf{D}}^{*}$. Note that $\psi_{(k)}\left(D_{\gamma_{j}}\right)=\mu_{j}$, for all $j=1, \ldots, n$.

Alternatively, if the second option of Lemma 10 holds, then we define a multiplicative functional $\psi_{\left\{m_{\ell}\right\}}$ on $\mathscr{A}_{\mathrm{D}}^{*}$ by

$$
\psi_{\left\{m_{\ell}\right\}}(D):=\lim _{\ell \rightarrow \infty}\left\langle D e_{\left(m_{\ell}, 0\right)}, e_{\left(m_{\ell}, 0\right)}\right\rangle .
$$

Lemma 11 (see [4, Lemma 4.12]). The limit (60) exists for all $D \in \mathscr{A}_{\mathrm{D}}^{*}$. The functional $\psi_{\left\{m_{e}\right\}}$ is multiplicative with $\psi_{\left\{m_{\ell}\right\}}\left(D_{\gamma_{j}}\right)=\mu_{j}$, for all $j=1, \ldots, n$.

Following [4] we give another formula defining the functional $\psi_{\left\{m_{\ell}\right\}}$ that permits an extension of $\psi_{\left\{m_{\ell}\right\}}$ to a larger subalgebra of $\mathscr{T}_{\text {rad }, q, k}$.

We start with the set $S=\left\{m_{\ell}: \ell \in \mathbb{N}\right\}$ and define the Hilbert space

$$
H_{S}=\overline{\operatorname{span}}\left\{e_{\alpha}: \alpha \in \mathbb{Z}_{+}^{2},|\alpha| \in S\right\} \subset \mathscr{A}_{\lambda}^{2}\left(\mathbb{B}^{2}\right) .
$$

The reproducing kernel $K_{S}$ of $H_{S}$ has the form

$$
K_{S}(z, w)=\sum_{|\alpha| \in S} e_{\alpha}(z) \overline{e_{\alpha}(w)} .
$$

Given $\zeta \in \bar{D}\left(0,\|q\|_{\infty}\right)$, let $\theta \in[0, \pi / 2], t_{1}, t_{2} \in \mathbb{T}$ be such that $\zeta=q(\theta)\left(t_{1} \overline{t_{2}}\right)^{k}$, and denote by $v=v\left(\theta, t_{1}, t_{2}\right)$ the corresponding point on $\partial \mathbb{B}^{2}$. Consider then a sequence $\left\{w_{h}\right\}_{h \in \mathbb{N}} \subset \mathbb{B}^{2}$ defined by $w_{h}=((h-1) / h) v$, and define the sequence $\left\{f_{h}\right\}_{h}$ of unit vectors in $H_{S}$ by

$$
f_{h}=\frac{K_{S}\left(\cdot, w_{h}\right)}{\left\|K_{S}\left(\cdot, w_{h}\right)\right\|} .
$$

Then, by [4, Lemma 4.13], the functional (60) can be also defined as

$$
\psi_{\left\{m_{e}\right\}}\left(D_{\gamma}\right)=\lim _{h \rightarrow \infty}\left\langle D_{\gamma} f_{h}, f_{h}\right\rangle .
$$

In order to extend the multiplicative functional (64) to a larger algebra we proceed as follows. Let $\zeta \in \bar{D}\left(0,\|q\|_{\infty}\right)$ and let $\theta \in[0, \pi / 2], t_{1}, t_{2} \in \mathbb{T}$ be such that $\zeta=q(\theta)\left(t_{1} \overline{t_{2}}\right)^{k}$. Then we take a sequence $\left\{w_{h}\right\}_{h}$ convergent to $v=v\left(\theta, t_{1}, t_{2}\right) \in \partial \mathbb{B}^{2}$ and $\left\{f_{h}\right\}_{h}$ as above.

Lemma 12 (see [4, Lemma 4.14]). The functional $\psi_{\left\{m_{\ell}\right\}}$ extends to the functional $\psi=\left(\psi_{\left\{m_{e}\right\}}, \zeta\right)$ on the algebra generated by elements of $\mathscr{A}_{\mathbf{D}}^{*}$ and $T_{q t^{*}}$ via

$$
\psi\left(D T_{q t^{\star}}^{j}\right)=\lim _{h \rightarrow \infty}\left\langle D T_{q^{\star}}^{j} f_{h}, f_{h}\right\rangle
$$

with $j \in \mathbb{Z}_{+}$and $D \in \mathscr{A}_{\mathbf{D}}^{*}$. Moreover, for elements of the form $\sum_{j=0}^{m} D_{j} T_{q t^{\kappa}}^{j}$ one has

$$
\psi\left(\sum_{j=0}^{m} D_{j} T_{q t^{\kappa}}^{j}\right)=\sum_{j=0}^{m} \psi_{\left\{m_{\ell}\right\}}\left(D_{j}\right) \zeta^{j} .
$$

4.2. Gelfand Theory. Recall that the commutative Banach algebra $\mathscr{T}_{\text {rad, } q, k}$ is generated by its two unital subalgebras $\mathscr{T}_{\text {rad }}$ and $\mathscr{T}_{q, k}$.

Recall also the following general fact: let $\mathscr{A}$ be a unital commutative Banach algebra generated by its two subalgebras $\mathscr{A}_{1}, \mathscr{A}_{2}$ sharing the same identity, and let $M(\mathscr{A}), M\left(\mathscr{A}_{1}\right)$, and $M\left(\mathscr{A}_{2}\right)$ be their respective sets of maximal ideals. Then we have a natural continuous mapping

$$
\iota: \psi \in M(\mathscr{A}) \longmapsto\left(\psi_{1}, \psi_{2}\right) \in M\left(\mathscr{A}_{1}\right) \times M\left(\mathscr{A}_{2}\right),
$$

defined by the restrictions $\psi_{1}$ and $\psi_{2}$ of the functional $\psi$ onto the subalgebras $\mathscr{A}_{1}, \mathscr{A}_{2}$. $M(\mathscr{A})$.

The mapping $\iota$ is injective identifying thus its range with

That is, by the results of Sections 3.1 and $3.2 \iota$ identifies $M\left(\mathscr{T}_{\text {rad }, q, k}\right)$ with a subset of $\left(\mathbb{Z}_{+} \cup M_{\infty}\right) \times \Delta(q, k)$.

Lemma 13. None of the points of the set $\mathbb{Z}_{+} \times(\Delta(q, k) \backslash\{0\})$ belongs to $M\left(\mathscr{T}_{\text {rad }, q, k}\right)$.

Proof. Suppose that $\psi=(m, \zeta) \in \mathbb{Z}_{+} \times(\Delta(q, k) \backslash\{0\})$ is an element of $M\left(\mathscr{T}_{\text {rad, } q, k}\right)$ and let $A=P_{m} T_{q^{*}} \in \mathscr{T}_{\text {rad, }, k}$ where $P_{m}$ is the orthogonal projection onto $H_{m}$; then $\psi(A)=1 \cdot \zeta \neq 0$ and, on the other hand, the operator $A$ belongs to the radical of $\mathscr{T}_{\text {rad } q, k}$; therefore $\psi(A)=0$ and we have a contradiction.

Note that the result of this lemma is independent of an extra condition (50) and thus from the concrete form of the spectrum $\Delta(q, k)$.

For the next result recall that the function $q$ satisfies condition (50), and thus $\Delta(q, k)=\bar{D}\left(0,\|q\|_{\infty}\right)$.

Lemma 14. One has that

(i) the set $\mathbb{Z}_{+} \times\{0\}$ belongs to $M\left(\mathscr{T}_{\text {rad }, q, k}\right)$,

(ii) the set $M_{\infty} \times \bar{D}\left(0,\|q\|_{\infty}\right)$ belongs to $M\left(\mathscr{T}_{\text {rad }, q, k}\right)$.

Proof. (i) Let $\psi=(m, 0) \in \mathbb{Z}_{+} \times\{0\}$. Let $\psi=(m, 0)=\left(\psi_{m}, 0\right)$ be a functional on a dense subalgebra $\mathscr{D}$ of $\mathscr{T}_{\text {rad }, q, k}$ defined, for any $A=\sum_{p=0}^{n} D_{p} T_{q t^{k}}^{p} \in \mathscr{D}$ where $D_{p} \in \mathscr{T}_{\text {rad }}$, by

$$
\begin{aligned}
\psi(A) & :=\left\langle A e_{(m, 0)}, e_{(m, 0)}\right\rangle \\
& =\psi_{(m)}\left(D_{0}\right) \\
& =\gamma_{D_{0}}(m),
\end{aligned}
$$

where $\psi_{(m)}$ is the multiplicative functional on $\mathscr{T}_{\text {rad }}$ given in (59). 
The functional $\psi$ is well defined, since $\sum_{p=0}^{n} D_{p} T_{q t^{\kappa}}^{p}=0$ implies $D_{0}=0$ by Lemma 6; moreover, $\psi$ is continuous and extends to a multiplicative functional on $\mathscr{T}_{\text {rad }, q, k}$ since

$$
\begin{aligned}
\left|\psi\left(\sum_{p=0}^{n} D_{p} T_{q t^{\kappa}}^{p}\right)\right| & =\left|\left\langle\sum_{p=0}^{n} D_{p} T_{q t^{\kappa}}^{p} e_{(m, 0)}, e_{(m, 0)}\right\rangle\right| \\
& \leq\left\|\sum_{p=0}^{n} D_{p} T_{q t^{\kappa}}^{p}\right\| .
\end{aligned}
$$

(ii) Let $\psi=(\mu, \zeta) \in M_{\infty} \times \bar{D}\left(0,\|q\|_{\infty}\right)$ and define a multiplicative functional on a dense subalgebra of $\mathscr{T}_{\text {rad,q,k }}$ as

$$
\psi\left(\sum_{p=0}^{n} D_{p} T_{q t^{\kappa}}^{p}\right):=\sum_{p=0}^{n} \mu\left(D_{p}\right) \zeta^{p}=\sum_{p=0}^{n} \gamma_{D_{p}}(\mu) \zeta^{p} .
$$

The functional $\psi$ is well defined:

$$
A=\sum_{p=0}^{n} D_{p} T_{q t^{\kappa}}^{p}=0
$$

implies that $D_{p}$ is compact for all $p=0, \ldots, n$ and since $\mu \epsilon$ $M_{\infty}, \mu\left(D_{p}\right)=0$ for all $p=0, \ldots, n$ we have that $\psi(A)=0$.

The functional $\psi$ is continuous: let $A=\sum_{p=0}^{n} D_{p} T_{q t^{\kappa}}^{p} \in$ $\mathscr{D}$ be a fixed element. Consider the unital $C^{*}$-algebra $\mathscr{A}_{\mathrm{D}}^{*}$ generated by $D_{0}, \ldots, D_{n}$. The restriction $\widehat{\psi}$ of $\psi$ to $\mathscr{A}_{\mathbf{D}}^{*}$ defines obviously a multiplicative functional on $\mathscr{A}_{\mathrm{D}}^{*}$. We note that

$$
\left(\mu_{0}, \ldots, \mu_{n}\right)=\left(\psi\left(D_{0}\right), \ldots, \psi\left(D_{n}\right)\right) \in \sigma\left(D_{0}, \ldots, D_{n}\right) .
$$

Since $\widehat{\psi}$ maps compact operators to zero, by (64), it has the form

$$
\widehat{\psi}\left(D_{\gamma}\right)=\lim _{h \rightarrow \infty}\left\langle D_{\gamma} f_{h}, f_{h}\right\rangle, \quad D_{\gamma} \in \mathscr{A}_{\mathbf{D}}^{*}
$$

where $\left\{m_{h}\right\}_{h} \subset \mathbb{Z}_{+}$is a suitable sequence induced by $\left(\mu_{0}, \ldots, \mu_{n}\right)$ as in Section 4.1 and $f_{h}$ is given by $(63)$.

By Lemma 12 we have

$$
\begin{aligned}
\left|\psi\left(\sum_{p=0}^{n} D_{p} T_{q t^{\kappa}}^{p}\right)\right| & =\lim _{h \rightarrow \infty}\left|\left\langle\sum_{p=0}^{n} D_{p} T_{q t^{\kappa}}^{p} f_{h}, f_{h}\right\rangle\right| \\
& \leq\left\|\sum_{p=0}^{n} D_{p} T_{q t^{\kappa}}^{p}\right\| ;
\end{aligned}
$$

therefore, $\psi$ is continuous and thus extends to a multiplicative functional on $\mathscr{T}_{\text {rad, } q, k}$.

Finally, Lemmas 13 and 14, Theorem 9, and properties of the injective tensor product imply the description of the set of maximal ideals and the Gelfand transform of Banach algebra $\mathscr{T}_{\operatorname{rad}, q, k}$.

Theorem 15. The compact set $M\left(\mathscr{T}_{\text {rad, } q, k}\right)$ of maximal ideals of the algebra $\mathscr{T}_{\text {rad,q,k }}$ has the form

$$
M\left(\mathscr{T}_{\operatorname{rad}, q, k}\right)=\mathbb{Z}_{+} \times\{0\} \cup M_{\infty} \times \bar{D}\left(0,\|q\|_{\infty}\right) .
$$

(i) The Gelfand image of the algebra $\mathscr{T}_{\mathrm{rad}, q, k}$ is isomorphic to $\mathscr{T}_{\mathrm{rad}, q, k} / \operatorname{Rad} \mathscr{T}_{\mathrm{rad}, q, k}$ and coincides with the algebra

$$
\operatorname{SO}\left(\mathbb{Z}_{+}\right) \cup\left[C\left(M_{\infty}\right) \widehat{\otimes}_{\epsilon} C_{a}\left(\bar{D}\left(0,\|q\|_{\infty}\right)\right)\right],
$$

which is identified with the set of all pairs

$$
(\gamma, f) \in S O\left(\mathbb{Z}_{+}\right) \times\left[C\left(M_{\infty}\right) \widehat{\otimes}_{\epsilon} C_{a}\left(\bar{D}\left(0,\|q\|_{\infty}\right)\right)\right]
$$

satisfying the following condition $\gamma(\mu)=f(\mu, 0)$ for all $\mu \in M_{\infty}$, where $\gamma(\mu)$ is identified with the value $\mu \in M_{\infty}$ on the element $\gamma \in S O\left(\mathbb{Z}_{+}\right)$.

The symbol $\widehat{\otimes}_{\epsilon}$ denotes the injective tensor product.

(ii) The Gelfand transform is generated by the following mapping of elements of $\mathscr{D}$ :

$$
\begin{aligned}
& \sum_{j=0}^{n} D_{\gamma_{j}} T_{q^{\kappa}}^{j} \\
& \qquad \begin{cases}\gamma_{0}(m), & \text { if }(m, 0) \in \mathbb{Z}_{+} \times\{0\}, \\
\sum_{j=0}^{n} \gamma_{j}(\mu) \zeta^{j}, & \text { if }(\mu, \zeta) \in M_{\infty} \times \bar{D}\left(0,\|q\|_{\infty}\right)\end{cases}
\end{aligned}
$$

\section{Conflict of Interests}

The authors declare that there is no conflict of interests regarding the publication of this paper.

\section{Acknowledgment}

The work was partially supported by the CONACYT Project 180049, Mexico.

\section{References}

[1] R. Quiroga-Barranco and N. Vasilevski, "Commutative $C^{*}$ algebras of TOeplitz operators on the unit ball. I. Bargmanntype transforms and spectral representations of TOeplitz operators," Integral Equations and Operator Theory, vol. 59, no. 3, pp. 379-419, 2007.

[2] R. Quiroga-Barranco and N. Vasilevski, "Commutative $C^{*}$ algebras of Toeplitz operators on the unit ball. II. Geometry of the level sets of symbols," Integral Equations and Operator Theory, vol. 60, no. 1, pp. 89-132, 2008.

[3] N. Vasilevski, "Quasi-radial quasi-homogeneous symbols and commutative Banach algebras of Toeplitz operators," Integral Equations and Operator Theory, vol. 66, no. 1, pp. 141-152, 2010.

[4] W. Bauer and N. Vasilevski, "On the structure of a commutative Banach algebra generated BY Toeplitz operators with quasiradial quasi-homogeneous symbols," Integral Equations and Operator Theory, vol. 74, no. 2, pp. 199-231, 2012.

[5] W. Bauer, C. Herrera, and N. Vasilevski, "Eigenvalue characterization of radial operators on weighted Bergman spaces over the unit ball," Integral Equations and Operator Theory, vol. 78, no. 2, pp. 271-300, 2014.

[6] R. Schmidt, "Über divergente Folgen und lineare Mittelbildungen," Mathematische Zeitschrift, vol. 22, no. 1, pp. 89-152, 1925. 
[7] T. W. Gamelin, Uniform Algebras, Prentice-Hall, Englewood Cliffs, NJ, USA, 1969.

[8] V. Rabinovich, S. Roch, and B. Silbermann, Limit Operators and Their Applications in Operator Theory, Birkhäuser, Basel, Switzerland, 2004.

[9] U. Venugopalkrishna, "Fredholm operators associated with strongly pseudo convex domains in $C^{n}$," Journal of Functional Analysis, vol. 9, pp. 349-373, 1972.

[10] W. Żelazko, "Continuous characters and joint topological spectrum," Control and Cybernetics, vol. 36, no. 3, pp. 859-864, 2007. 


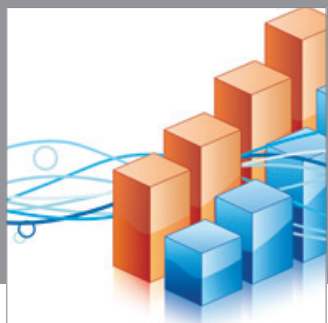

Advances in

Operations Research

mansans

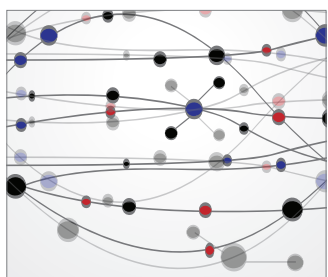

The Scientific World Journal
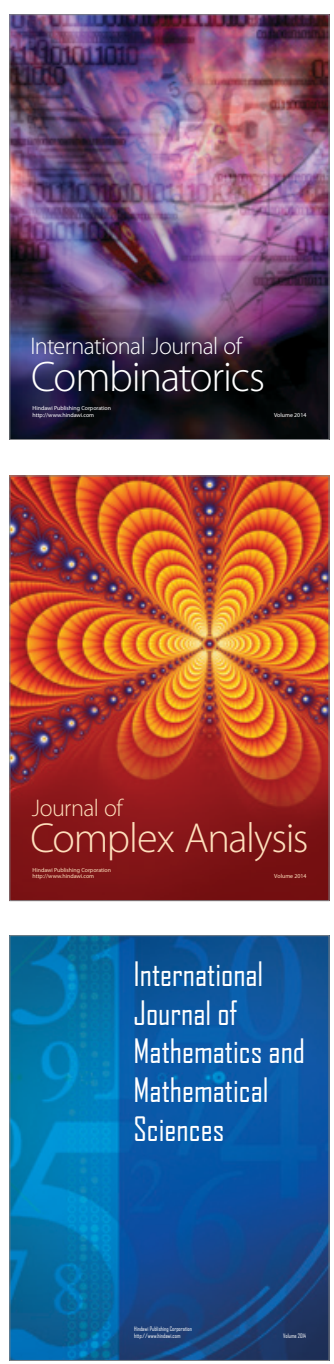
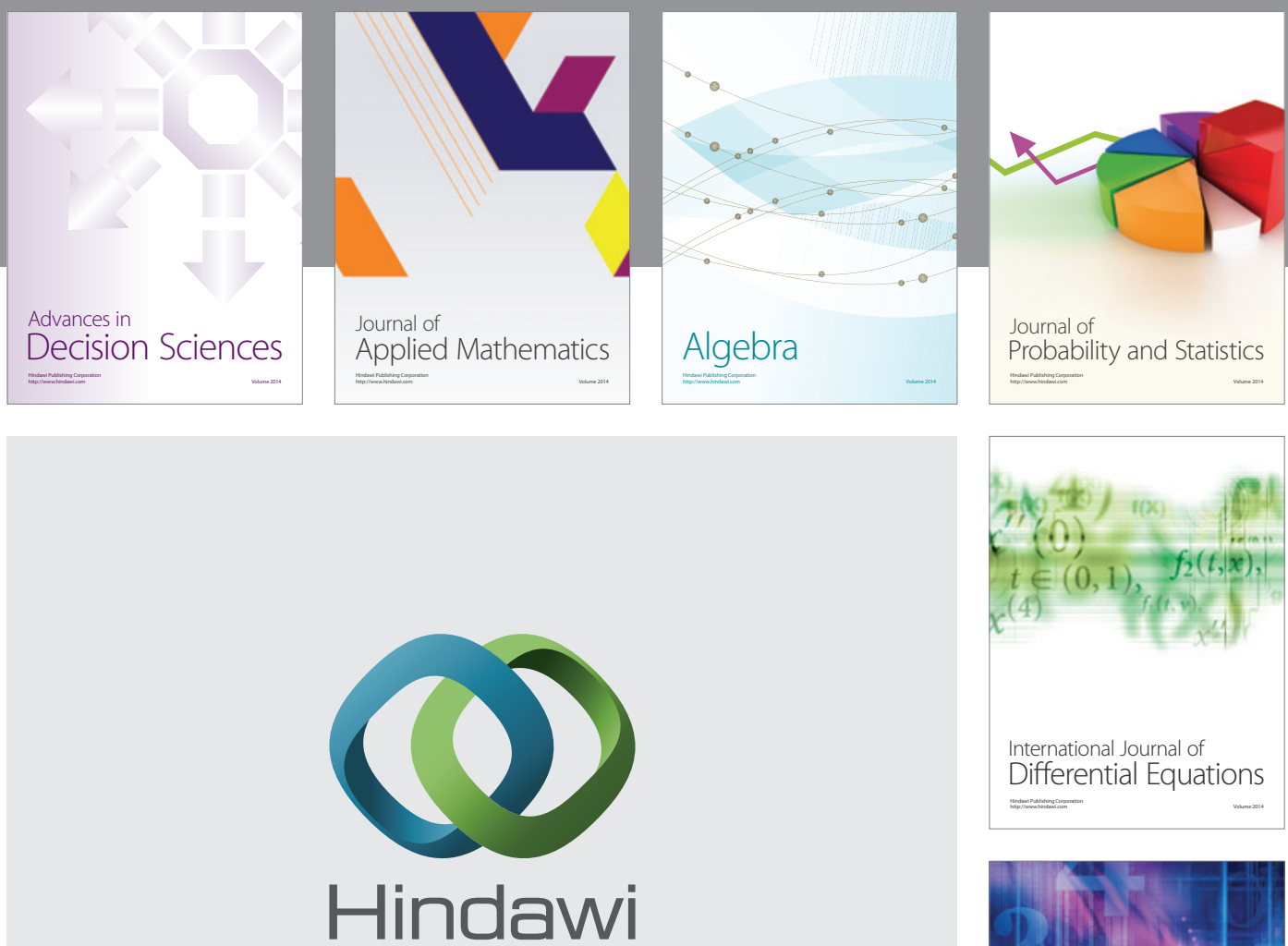

Submit your manuscripts at http://www.hindawi.com
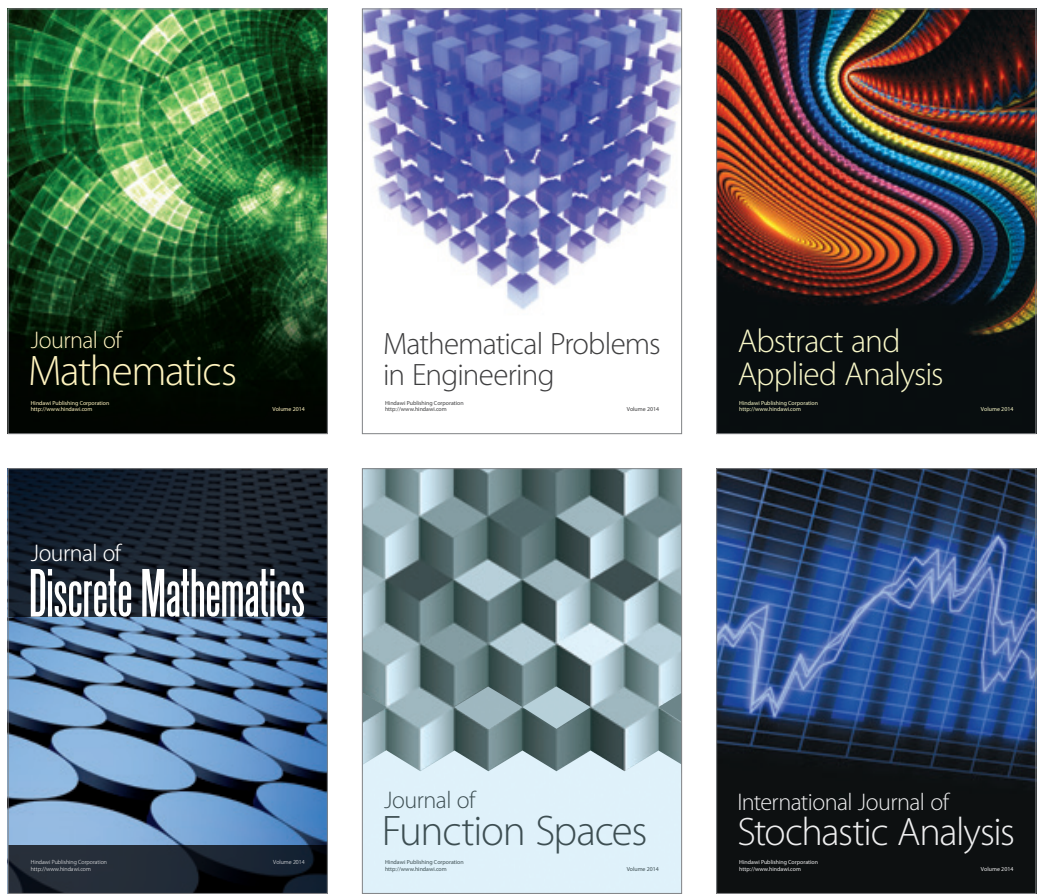

Journal of

Function Spaces

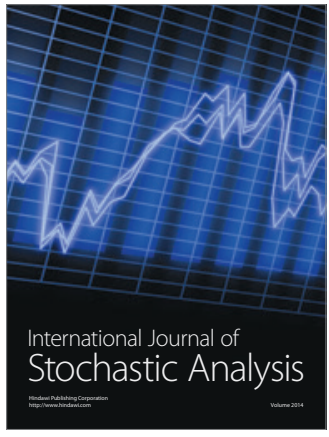

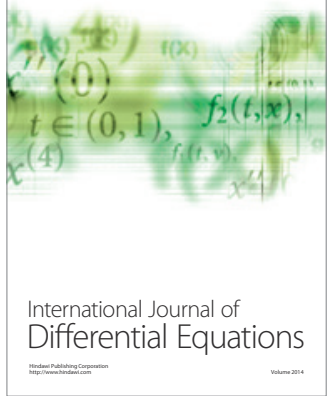
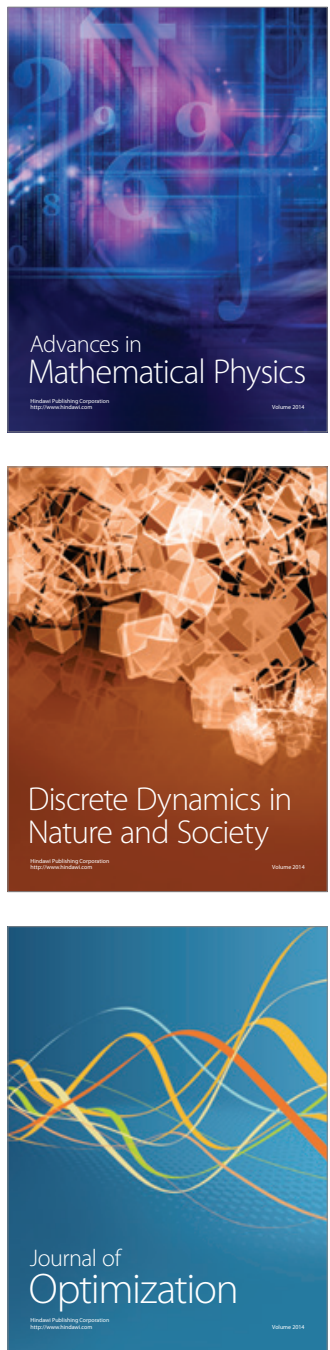